\title{
On the Representation of Heterogeneity in Land-Surface-Atmosphere Coupling
}

\author{
Philipp de Vrese ${ }^{1} \cdot$ Jan-Peter Schulz ${ }^{2}$. \\ Stefan Hagemann ${ }^{1}$
}

Received: 3 February 2015 / Accepted: 2 February 2016 / Published online: 17 February 2016

(C) The Author(s) 2016. This article is published with open access at Springerlink.com

\begin{abstract}
A realistic representation of processes that are not resolved by the model grid is one of the key challenges in Earth-system modelling. In particular, the non-linear nature of processes involved makes a representation of the link between the atmosphere and the land surface difficult. This is especially so when the land surface is horizontally strongly heterogeneous. In the majority of present day Earth system models two strategies are pursued to couple the land surface and the atmosphere. In the first approach, surface heterogeneity is not explicitly accounted for, instead effective parameters are used to represent the entirety of the land surface in a model's grid box (parameter-aggregation). In the second approach, subgridscale variability at the surface is explicitly represented, but it is assumed that the blending height is located below the lowest atmospheric model level (simple flux-aggregation). Thus, in both approaches the state of the atmosphere is treated as being horizontally homogeneous within a given grid box. Based upon the blending height concept, an approach is proposed that allows for a land-surface-atmosphere coupling in which horizontal heterogeneity is considered not only at the surface, but also within the lowest layers of the atmosphere (the VERTEX scheme). Below the blending height, the scheme refines the turbulent mixing process with respect to atmospheric subgrid fractions, which correspond to different surface features. These subgrid fractions are not treated independently of each other, since an explicit horizontal component is integrated into the turbulent mixing process. The scheme was implemented into the JSBACH model, the land component of the Max Planck Institute
\end{abstract}

Electronic supplementary material The online version of this article (doi:10.1007/s10546-016-0133-1) contains supplementary material, which is available to authorized users.

$凶 \quad$ Philipp de Vrese

philipp.de-vrese@mpimet.mpg.de

Jan-Peter Schulz

jan-peter.schulz@dwd.de

Stefan Hagemann

stefan.hagemann@mpimet.mpg.de

1 Max Planck Institute for Meteorology, Bundesstrae 53, 20146 Hamburg, Germany

2 Deutscher Wetterdienst, Frankfurter Strasse 135, 63067 Offenbach, Germany 
for Meteorology's Earth-system model, when coupled to the atmospheric general circulation model ECHAM. The single-column version of the Earth system model is used in two example cases in order to demonstrate how the effects of surface heterogeneity are transferred into the atmosphere, influencing local stability and the turbulent mixing process. Furthermore, a simple flux-aggregation scheme was implemented into the JSBACH model. By comparing single-column simulations utilizing the VERTEX scheme and the simple flux-aggregation scheme, it can be shown that the horizontal disaggregation of the turbulent mixing process has a substantial impact on the simulated mean state of a grid box. Here, the differences between simulations with the two schemes may, in certain cases, be even larger than the differences between simulations with the simple flux-aggregation scheme and simulations in which surface heterogeneity is not explicitly accounted for (i.e., a parameter-aggregation scheme).

Keywords Flux aggregation · Surface heterogeneity · Tile approach · Turbulent mixing

\section{Introduction}

Representing spatially heterogeneous processes, taking place on scales below those resolved by present day numerical models, is one of the key challenges in Earth-system modelling. Here, one aspect is to accurately describe the interaction between the land surface and the atmosphere. Various land-surface characteristics such as topography, land use, soil and vegetation characteristics vary on diverging spatial scales and time scales, ranging from a few fractions of a metre and minutes to hundreds of kilometres and years. Consequently, the state of the surface varies on the same scales. Surface energy, mass and momentum fluxes physically link the surface and the atmosphere and often have a highly non-linear dependency upon this horizontally strongly heterogeneous state of the land surface (Sellers 1991). Thus, considering the range of scales that needs to be accounted for reveals the difficulties that occur when attempting to represent the affected processes on the grid scale of an Earth-system model.

In contemporary Earth-system models, different strategies are pursued to describe the contribution of subgrid-scale (SGS) variability and to aggregate the SGS information of the land surface to match the grid of the atmosphere. Due to the non-linear nature of processes involved, every strategy results in a distinct representation of the land-surface-atmosphere interaction. The nomenclature in respect to these strategies and their various implementations is confusing at best. Hence, a few definitions of terms that will be used in this work are given below.

One general distinction between strategies can be made between those based on statistical and those based on discrete representations of SGS heterogeneity (Giorgi and Avissar 1997). In methods belonging to the first group, SGS variability is represented by a variable specific probability density function, and the aggregation of the SGS parameter values is performed by integrating over the parameter phase space defined by the respective probability density function. In this work, the focus is placed on discrete representations of SGS heterogeneity and no further discussion of the statistical approach will follow. A good overview on this approach can be found, for example, in Avissar (1992).

The basic assumption of the discrete approaches is that a grid box can be sectioned into discrete sub-divisions, the so-called "tiles" or "patches", which themselves exhibit homogeneous characteristics. These are usually represented simply by their cover fraction, i.e., 
the fraction of the grid-box area that they occupy, ignoring the actual geographical location. However, methods exist in which the tiles represent clearly defined areas with a specific size and position within the grid box (Seth et al. 1994). The discrete schemes further divide into two major branches. In one branch, SGS heterogeneity is not explicitly accounted for, but soil and vegetation parameters of the SGS patches are aggregated to one effective value, which represents the entire grid box. Based upon these effective values the surface fluxes are calculated; herein this will be referred to as the "parameter-aggregation". The most common approach to determine the effective grid parameters is by averaging all SGS parameter values usually weighted by their respective cover fractions. Another possibility, the so-called "dominant approach", is to represent a grid box by the characteristics of the dominant tile, i.e., the one that covers the largest fraction of the grid box (Dickinson 1986). In the following, the term parameter-aggregation exclusively refers to the aggregation based upon the area-weighted average.

The second branch explicitly accounts for SGS heterogeneity and calculates fluxes based upon the tile specific characteristics for all tiles in a grid box individually. Here, two approaches can be distinguished that Koster and Suarez (1992) define as the "mosaic" and the "mixture approach". The two approaches differ in the assumptions made about the extent to which the individual tiles interact. Note that the use of terms substantially varies between studies. For instance, Ament and Simmer (2006) use a distinction, which is based upon the representation of the sub-divisions either as a localized patch in the grid box or by their cover fraction to distinguish between the mosaic and a tile approach. In contrast to the use of terms of Koster and Suarez (1992), Molod and Salmun (2002) use the term mixture approach for a form of parameter-aggregation.

The mosaic approach assumes that the different tiles or patches in one grid box are horizontally well separated and that, below the lowest computational level in the atmospheric model, the horizontal exchange between tiles is negligible in comparison to the vertical fluxes. Consequently, the interaction of each surface tile and the overlying atmosphere is completely independent of the other tiles present in the grid box. In the classical mosaic approach, the assumption of the independence of each tile is valid only for the surface layer, and higher up all processes, including the turbulent vertical fluxes, are modelled based upon the grid mean state (Avissar and Pielke 1989; Giorgi 1997). However, the mosaic approach has been augmented to preserve the independence of tiles throughout the entire planetary boundary layer (PBL) at least for the vertical turbulent transport (Molod et al. 2003).

In contrast to this, the mixture or tile approach assumes that surface heterogeneity occurs in the form of numerous small clusters. These clusters are evenly distributed across the grid box, and the horizontal turbulent fluxes are large compared to the vertical turbulent fluxes. Therefore, the properties of air parcels originating from different tiles are perfectly mixed horizontally, even below the lowest atmospheric model level, and the atmosphere interacts only with a horizontally homogeneous flux (Koster and Suarez 1992). Consequently, the state of the atmosphere is spatially homogeneous within a grid box. Both mosaic and mixture approach are often referred to as flux-aggregation techniques, however in the present study the term "simple flux-aggregation" is reserved for the mixture or tile approach.

Many observational and modelling studies suggest that both of these underlying assumptions are erroneous under certain conditions. The signal related to a specific surface heterogeneity may be detectable up to a given height within the PBL that lies far above the surface layer. This indicates that the assumption of a horizontally homogeneous flux below the lowest atmospheric model level (in many models this is below $50 \mathrm{~m}$, see Arola 1999 ) is not valid in the respective conditions. In different conditions, fluxes may not be in equilibrium with the local surface even low within the surface layer, indicating that the 
horizontal turbulent transport processes have a noticeable impact and are not negligible. This rebuts the assumption of the independence of tiles made in the mosaic approach (Wieringa 1976; Mason 1988; Claussen 1995; Raupach and Finnigan 1995; Avissar and Schmidt 1998; Mahrt 2000; Bou-Zeid et al. 2004; Patton et al. 2005; Ma et al. 2008).

In the following, a technique is proposed that supersedes the need for potentially invalid simplifications when coupling land surface and atmosphere. Therefore, when implemented in a land-surface model, this technique could improve the aggregation of SGS information for the coupling to an atmospheric general circulation model (GCM). This coupling scheme, which provides a VERtical Tile EXtension (VERTEX), is capable of representing the turbulent mixing process more realistically, as it resolves the process with respect to the surface tiles, while explicitly accounting for the horizontal component of the process. Thus, within a model grid box the turbulent transport is treated as a two-dimensional process (vertical and horizontal) rather than a one-dimensional process (vertical only). To minimize confusion, the horizontal component of the mixing process, which homogenises the state and the vertical fluxes within the grid box, is referred to as blending.

In Sect. 2, the VERTEX scheme is introduced. Firstly (Sect. 2.1), we discuss how the horizontal component of the turbulent mixing process, i.e., the horizontal blending of air properties and the vertical fluxes between the tiles within a grid box, can be related to the blending height concept (see e.g., Mahrt 2000). In Sects. 2.2 and 2.3, a concept is presented to integrate an explicit horizontal component into an atmospheric model's (vertical) turbulent exchange scheme. This includes closing the surface energy balance, based on the assumption of a horizontally varying state of the lowest atmospheric model levels within a model grid box. In the subsequent section, the VERTEX scheme is employed in two example cases in order to demonstrate its impact on the simulated structure of the atmosphere (Sects. 3.1 and 3.2).

Additionally, we compare results of simulations with the different flux-aggregation schemes, to gain further insights into the mechanisms and the magnitude of potential impacts on the simulated mean state of a grid box (Sect. 3.3). Many studies exist, usually focused on the local scale, which have compared parameter aggregating to flux-aggregating techniques (see e.g., Avissar and Pielke 1989; Polcher et al. 1996; Van den Hurk and Beljaars 1996; Cooper et al. 1997; Molod and Salmun 2002; Essery et al. 2003; Heinemann and Kerschgens 2005; Ament and Simmer 2006). These studies agree that by employing an aggregation of fluxes the representation of processes clearly improves compared to an aggregation of parameters. In addition to this, many of the studies find an improvement in the simulated climate. Thus, we also compare the simulations performed with the different flux-aggregation methods to simulations, which are carried out using a parameter-aggregation scheme, and use these comparisons as a reference for the magnitude of potential impacts. Finally, main results are summarized in Sect. 4.

\section{The VERTEX Scheme}

\subsection{Blending Height and Horizontal Mixing}

Studies investigating the vertical extent to which the impact of surface heterogeneity is detectable often employ the concept of a blending height. The blending height can be understood as a scaling depth that "describes the decrease of the influence of surface heterogeneity with height" below a certain threshold (Mahrt 2000). In reverse this means that below the 
blending height the influence of individual surface features is perceptible. Hence, the blending height can also be interpreted as the maximum distance that a surface feature perceptibly exerts its influence in the vertical direction.

The blending height $h_{\text {blend, } i}$ can be expressed as a function of the friction velocity $u_{*, i}$, the horizontal wind speed $U$ at the blending height, the characteristic length scale $L_{\text {hetero, } i}$ of a given surface feature $i$, and the two non-dimensional coefficients $C$ and $p$ (Raupach and Finnigan 1995; Mahrt 2000),

$$
h_{\text {blend }, i}=C\left(\frac{u_{*, i}}{U}\right)^{p} L_{\text {hetero }, i} .
$$

Equation 1 gives the general form of expressing the blending height, applicable in near-neutral or stable conditions, when shear-driven turbulence predominates. In the case of the convective boundary-layer (CBL) turbulence is largely buoyancy driven. Under unstable conditions, the dependence of the blending height on instability (given by $u_{*, i} / U$ ) may be too weak, and a formulation that accounts for a more explicit dependence on instability may be required. For a "thermal blending height", Wood and Mason (1991) suggest an approximation that is based on $U, L_{\text {hetero, } i}$, the spatially-averaged surface sensible heat flux $H_{0}$, the spatially-averaged potential temperature $\theta_{0}$ and the coefficient $C_{\text {therm }}$,

$$
h_{\text {blend }, i}=C_{\text {therm }}\left(\frac{H_{0}}{U \theta_{0}}\right) L_{\text {hetero }, i} .
$$

By assuming a fixed relation between height and the magnitude of the influence that a heterogeneity exerts on the flow at a height $h_{z}$, one can relate the ratio of a given height and the blending height to the strength of the surface heterogeneity's influence. By further assuming that the decrease of the influence of a surface feature with height can be attributed to the horizontal exchange of air properties one can describe $d_{z, i}$, the degree to which the heterogeneous vertical fluxes have blended at a given $h_{z}$ above a surface feature $i$, by $h_{\text {blend, } i}$ and $h_{z}$,

$$
d_{z, i}=f\left(h_{z}, h_{\text {blend }, i}\right)
$$

for $0 \leq h_{z} \leq h_{\text {blend, } i}$. Furthermore, in a grid box containing $n$ tiles, $m_{z, i, j}$, the proportion of the vertical flux above surface feature $i$ at height $h_{z}$ that is determined by the characteristics of a given surface feature $j$ with the cover fraction $c f_{j}$, is given by

$$
m_{z, i, j}=d_{z, i} \frac{d_{z, j} c f_{j}}{\sum_{k=1}^{n} d_{z, k} c f_{k}}
$$

for $j \neq i$,

$$
m_{z, i, j}=\left(1-d_{z, i}\left(1-\frac{d_{z, j} c f_{j}}{\sum_{k=1}^{n} d_{z, k} c f_{k}}\right)\right)
$$

for $j=i$. The relation between the terms used in Eq. 4 is summarized in Fig. 1 .

So far the approach accounts only for the direct "aggregation" effects related to surface heterogeneity and neglects "dynamical" effects that are associated with SGS circulations (Molod et al. 2003). A non-uniform heating of the surface results in a non-uniform pressure distribution. Due to this spatial pressure variability, baroclinic circulations develop, which reduce strong spatial gradients. For low horizontal model resolutions, i.e., a typical GCM grid spacing $>100 \mathrm{~km}$, these mesoscale circulations become increasingly important. There are indications that organized mesoscale circulations predominantly occur for length scales of $L_{\text {hetero }}>10 \mathrm{~km}$ (Baidya Roy 2003). However, there is observational evidence for atmospheric 


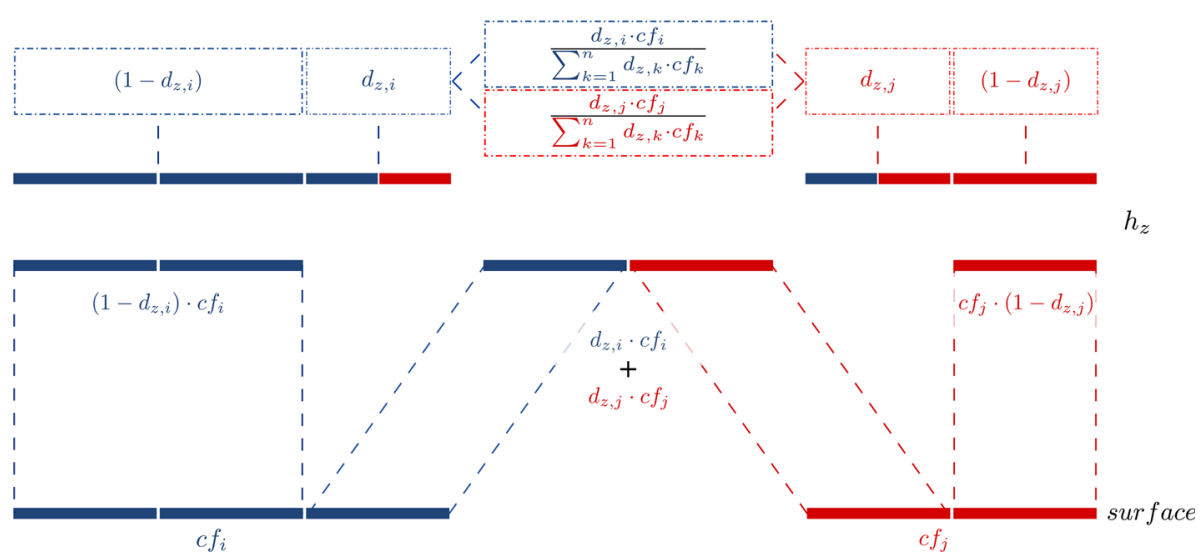

Fig. 1 Terms constituting $m$ in Eq. 4 in the case of two differing surfaces (blue and red)

spatial heterogeneity related to surface heterogeneity with $L_{\text {hetero }}>10 \mathrm{~km}$ (Segal et al. 1989; Angevine 2003; Banta 2003; Strunin et al. 2004). This suggests that, while mesoscale circulations have a balancing effect, spatially heterogeneous atmospheric structures prevail even at these scales.

In general, the concept of the tiling method does not allow to represent SGS circulations explicitly, as it does not provide any information on the relative position of the tiles within the grid box. Nonetheless, it is feasible to parametrize unresolved circulations by considering them in the formulation of the horizontal blending of vertical fluxes, i.e., in the calculation of $d_{z, i}$ and $m_{z, i, j}$ (Lynn et al. 1995a, b). For example, by assuming that mesoscale circulations act to reduce strong spatial gradients, $d_{z, i}$ could be altered for large differences between $T_{\text {surf }, i}$, the surface temperature in tile $i$, and $\bar{T}_{\text {surf }}$, the grid-box mean temperature, or $m_{z, i, j}$ could be adjusted for temperature differences between tile $i$ and $j$.

$$
d_{z, i}=f\left(\left|T_{\text {surf }, i}-\bar{T}_{\text {surf }}\right|, h_{z}, h_{\text {blend }, i}\right)
$$

for $0 \leq h_{z} \leq h_{\text {blend }, i}$,

$$
m_{z, i, j}=f\left(\left|T_{\text {surf }, i}-T_{\text {surf }, j}\right|, d_{z, 1}, c f_{1}, \ldots, d_{z, n}, c f_{n}\right) .
$$

Modelling the horizontal component of the turbulent mixing process requires quantifying the strength of the horizontal component of the process between two given vertical model levels $l-1$ and $l$. To calculate $m_{l, i, j}$, the degree to which the fluxes from a given tile $j$ and the tile $i$ blend between the levels $l-1$ and $l$, the following equation can be used,

$$
m_{l, i, j}=(1-\epsilon) \frac{d_{l, j} c f_{j}}{\sum_{k=1}^{n} d_{l, k} c f_{k}}
$$

for $j \neq i$,

$$
m_{l, i, j}=\epsilon+(1-\epsilon) \frac{d_{l, j} c f_{j}}{\sum_{k=1}^{n} d_{l, k} c f_{k}}
$$

for $j=i$, with

$$
\epsilon=\frac{1-d_{l, i}}{1-d_{l-1, i}}
$$


In the formulation, the levels $l-1$ and $l$ correspond to two given heights $z-\delta z$ and $z$ above the surface. The vertical fluxes at level level $l-1$ have already started to become horizontally homogeneous, and $m_{l, i, j}$ accounts only for the additional horizontal blending that occurs between the levels $l-1$ and $l$. With the help of these blending coefficients, the turbulent mixing process can be represented as a two-dimensional process, which is described in detail below.

\subsection{Vertical Diffusion and Horizontal Mixing}

The (vertical) turbulent mixing process of humidity, sensible heat and momentum is often described by

$$
\frac{\partial x}{\partial t}=\frac{\partial}{\partial z}\left(K \frac{\partial x}{\partial z}\right)
$$

where $K\left[\mathrm{~m}^{2} \mathrm{~s}^{-1}\right]$ denotes the vertical eddy diffusivity, and $x$ is the transported quantity. The transport of momentum can be modelled the same way as that of heat and moisture. However, we limit the following explanation to the latter two, and $x$ represents either dry static energy or specific humidity.

For a horizontally homogeneous grid box, this can be written in a vertically discretized way, using an implicit time-stepping. The rate of change of humidity or sensible heat can be expressed at a given level $l$ by

$$
\frac{x_{l}^{t+1}-x_{l}^{t}}{\Delta t}=\frac{1}{\Delta z_{l}}\left(\Theta X_{l}-\Theta X_{l-1}\right),
$$

with

$$
\Theta X_{l}=K_{l+\frac{1}{2}} \frac{x_{l+1}^{t+1}-x_{l}^{t+1}}{\delta z_{l}},
$$

and

$$
\Theta X_{l-1}=K_{l-\frac{1}{2}} \frac{x_{l}^{t+1}-x_{l-1}^{t+1}}{\delta z_{l-1}},
$$

where $\Delta z_{l}$ pertains to the thickness of a layer encompassed by two full vertical model levels on which the variables are calculated, whereas $\delta z_{l}$ pertains to a layer that is bounded by two intermediate levels on which the fluxes are calculated (see e.g., Polcher et al. (1998)). Following Richtmyer and Morton (1967), this set of equations can be solved for the entire atmosphere at timestep $t+1$. At the top of the atmospheric column $(l=N)$ the fluxes are assumed to be zero. This allows the top level dry static energy (specific humidity) to be described by a function of the dry static energy (specific humidity) on the level below and the two coefficients $E_{x, l-1}^{t}$ and $F_{x, l-1}^{t}$,

$$
x_{l}^{t+1}=E_{x, l-1}^{t} x_{l-1}^{t+1}+F_{x, l-1}^{t},
$$

where $E_{x, l-1}^{t}$ and $F_{x, l-1}^{t}$ at the top of the atmosphere are determined by Eq. 8 (for $l=N$ ) applying the zero flux condition.

When the $E_{x, l}^{t}$ and $F_{x, l}^{t}$ are known for the superjacent level, this process can be repeated for each level in a descending order from $l=N-1$ to the surface $(l=0)$. Here, the surface energy balance is solved, which yields the dry static energy and humidity at the surface at timestep $t+1$. Having determined the surface dry static energy and humidity, these can be 


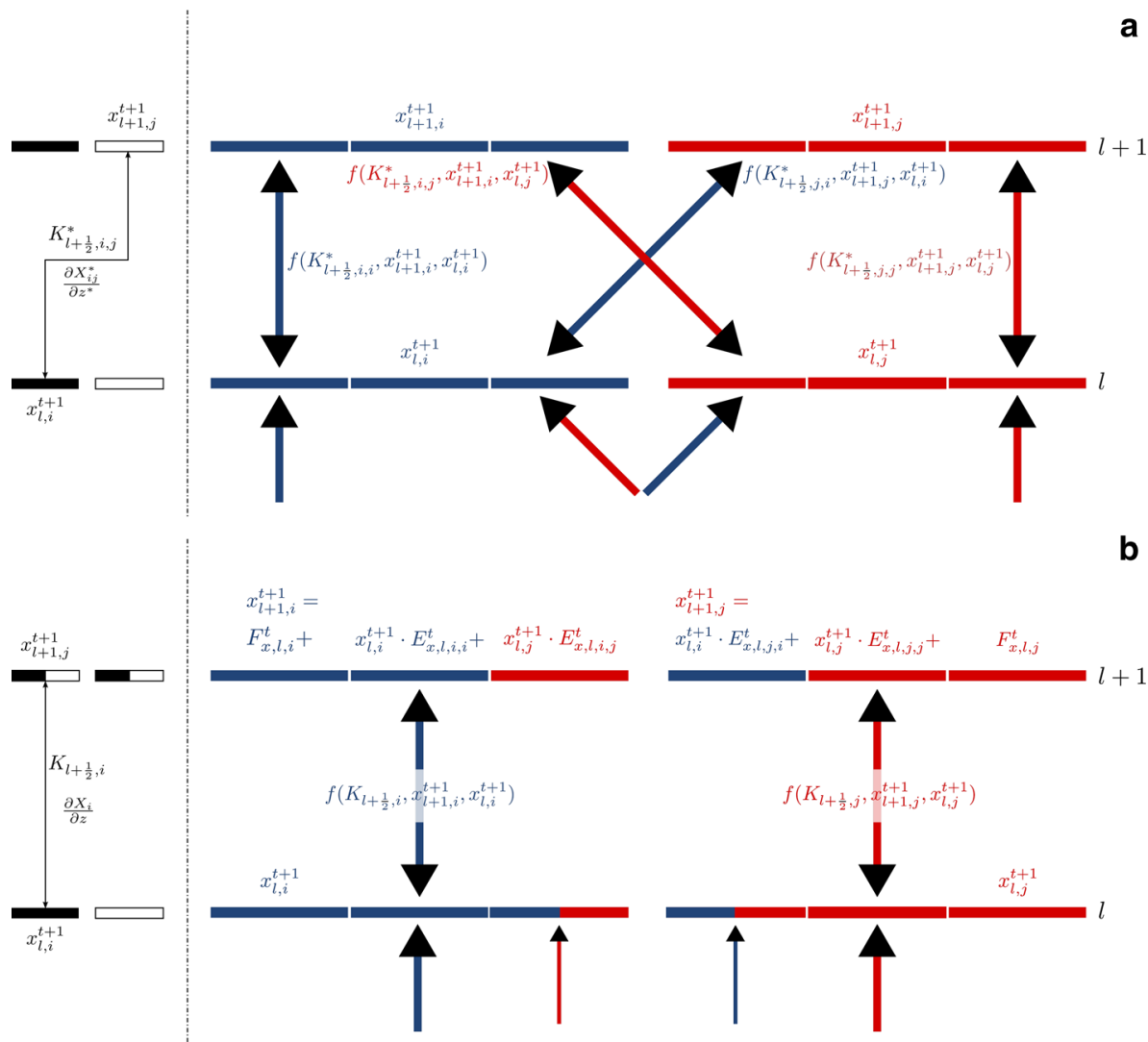

Fig. 2 Simplifying assumptions for the vertical turbulent transport: a Terms used in Eq. 11, b Terms used in Eq. 12, substituting $x_{l+1, i}^{t+1}$ and $x_{l+1, j}^{t+1}$ with Eq. 13

used in a back-substitution to calculate the new values for the entire atmospheric column using Eq. 9 (Schulz et al. 2001).

In the case of a horizontally heterogeneous grid box, Eq. 7 needs to be augmented to allow for different tiles and a turbulent mixing process, which consists of not only a vertical component, but also a quasi-horizontal component, i.e., the blending of fluxes from the different tiles

$$
\frac{\partial X_{i}}{\partial t}=\frac{\partial}{\partial z}\left(\sum_{j=1}^{n} m_{z, i, j} K_{i j}^{*} \frac{\partial X_{i j}^{*}}{\partial z^{*}}\right),
$$

where $\partial X_{i j}^{*} / \partial z^{*}$ denotes the gradient of $X_{i j}^{*}$ between two heights $z_{1}$ and $z_{2}$ and between two tiles $i$ and $j, K_{i j}^{*}$ is the eddy diffusivity between $z_{1}$ and $z_{2}$ and between the tiles $i$ and $j$, and $m_{z, i, j}$ is the degree to which the fluxes in tile $i$ have blended with the fluxes from tile $j$ (Fig. 2).

The discretization of Eq. 10 in the vertical and in time yields a representation of the turbulent mixing process, which takes into account a quasi-horizontal, inter-tile component. 


$$
\frac{x_{l, i}^{t+1}-x_{l, i}^{t}}{\Delta t}=\frac{1}{\Delta z_{l}}\left(\sum_{j=1}^{n} \Theta X_{l, j, i}^{*}-\Theta X_{l-1, i, j}^{*}\right),
$$

with

$$
\Theta X_{l, j, i}^{*}=m_{l, j, i} K_{l+\frac{1}{2}, j, i}^{*} \frac{x_{l+1, j}^{t+1}-x_{l, i}^{t+1}}{\delta z_{l}},
$$

and

$$
\Theta X_{l-1, i, j}^{*}=m_{l-1, i, j} K_{l-\frac{1}{2}, i, j}^{*} \frac{x_{l, i}^{t+1}-x_{l-1, j}^{t+1}}{\delta z_{l-1}} .
$$

In order to be able to solve the set of equations analogous to the case of a horizontally homogeneous grid box, further simplifying assumptions are required (Fig. 2):

- for a given level $l$ and tile $i$, the tile specific flux connecting level $l$ to the level above $(l+1)$ is determined exclusively by the vertical gradient of $x$ within the respective tile $i$,

- whereas the flux connecting level $l$ to the subjacent level $l-1$ is a weighted average of the tile individual fluxes, as the fluxes from different tiles $(1, \ldots, j, \ldots, n)$ have blended to a certain degree (given by $m_{l-1, i, j}$ ).

These simplifications allow Eq. 11 to be written using simple vertical gradients of $x$ and the vertical eddy diffusivity $K$ instead of $K^{*}$,

$$
\frac{x_{l, i}^{t+1}-x_{l, i}^{t}}{\Delta t}=\frac{1}{\Delta z_{l}}\left(\Theta X_{l, i}-\sum_{j=1}^{n} \Theta X_{l-1, i, j}\right),
$$

with

$$
\Theta X_{l, i}=K_{l+\frac{1}{2}, i} \frac{x_{l+1, i}^{t+1}-x_{l, i}^{t+1}}{\delta z_{l}},
$$

and

$$
\Theta X_{l-1, i, j}=m_{l-1, i, j} K_{l-\frac{1}{2}, j} \frac{x_{l, j}^{t+1}-x_{l-1, j}^{t+1}}{\delta z_{l-1}} .
$$

The concept of solving the vertical diffusion scheme remains to describe the dry static energy (specific humidity) on a given level as a function of the dry static energy (specific humidity) on the subjacent level. However, when considering a given tile within a horizontally heterogeneous grid box, the formulation has not only to include the same tile on the level below, but all the tiles within the grid box,

$$
x_{l+1, i}^{t+1}=F_{x, l, i}^{t}+\sum_{j=1}^{n} x_{l, j}^{t+1} E_{x, l, i, j}^{t} .
$$

This formulation can be inserted into Eq. 12, and the resulting set of equations can be subsequently solved from the first level above the blending height until the second lowest atmospheric model level. The system of representing the state on a given atmospheric level by the state on the subjacent level also provides the basis for the simplifying assumptions used in the scheme. For level $l$ and tile $i$, it is possible to describe the fluxes between level $l$ and the superadjacent level $l+1$ simply by the local gradient of $x_{i}$, because the upper boundary 
used to calculate the fluxes, i.e., $x_{l+1, i}$, already includes the dependencies on the states of all the tiles on level $l$ (Fig. 2). As the formulation of the coefficients $F_{x, l, i}^{t}$ and $E_{x, l, i, j}^{t}$ for each level (level $l$ ) is based upon the right-hand side of Eq. 12 in respect to the level above $($ level $l+1)$, the scheme is conservative in terms of the transported variables. Here, Eq. 11 and Eq.12 (when substituting $x_{l+1, i}^{t+1}$ with Eq.13) yield only minor differences, except during the transition between stable and unstable stratification. In these circumstances, it is conceivable that $x_{l+1, i}^{t} \approx x_{l, i}^{t}$ and $x_{l+1, j}^{t} \approx x_{l, j}^{t}$, hence $K \approx 0$, but $x_{l+1, i}^{t} \neq x_{l, j}^{t}$ and $x_{l+1, j}^{t} \neq x_{l, i}^{t}$, hence $K^{*}>0$. However, a preliminary investigation indicated that this results in differences in the exact timestep in which the transition occurs, but does not cause large or lasting differences between the simulated states of the tiles.

To describe the dry static energy and specific humidity on the lowest atmospheric level, the surface fluxes have to be utilized. This constitutes the link between the surface and the atmosphere.

\subsection{Surface Fluxes and the Surface Energy Balance}

In order to couple the atmosphere to the land surface, a relation between the energy and moisture fluxes at the surface and the values of dry static energy and specific humidity at the lowest atmospheric model level is required (Best et al. 2004). The same simplifications that are applied higher up in the atmosphere are also applied to the surface fluxes. Conceptually this means that a distinction is made between considering the fluxes just above the surface $Q_{x, j}^{t+1, *}$ and considering them just below the lowest atmospheric model level $Q_{x, i}^{t+1}$. At the surface, the tile specific fluxes are assumed to depend exclusively on local surface-atmosphere differences and can be calculated according to

$$
\begin{aligned}
H_{i}^{t+1 *} & =c_{\mathrm{cp}, i}\left(s_{1, i}^{t+1}-s_{\text {surf }, i}^{t+1}\right), \\
L E_{i}^{t+1 *} & =c_{\mathrm{cp}, i} L \beta_{i}\left(q_{1, i}^{t+1}-\alpha_{i} q_{\mathrm{sat}}\left(c_{p}^{-1} s_{\mathrm{surf}, i}^{t+1}\right)\right),
\end{aligned}
$$

where $c_{\mathrm{cp}, i}$ is the product of the surface drag coefficient and the horizontal wind speed, $L$ is the latent heat of vaporization, $\alpha_{i}$ and $\beta_{i}$ are parameters that control evapotranspiration with respect to the availability of water.

At the lowest atmospheric level, the fluxes have blended to a certain degree, and the energy and moisture fluxes at the lowest atmospheric level $Q_{x, i}^{t+1}$ can be described by the tile specific fluxes just above the surface $Q_{x, j}^{t+1, *}$ and the blending coefficients $m_{1, i, j}$ described in Sect. 2.1,

$$
Q_{x, i}^{t+1}=\sum_{j=1}^{n} m_{1, i, j} Q_{x, j}^{t+1, *}
$$

The rate of change of dry static energy (specific humidity) on the lowest atmospheric level can be described by

$$
\frac{x_{1, i}^{t+1}-x_{1, i}^{t}}{\Delta t}=\frac{1}{\Delta z_{l}}\left(K_{1+\frac{1}{2}, i} \frac{F_{1, i}^{t}+\sum_{j=1}^{n} x_{1, j}^{t+1} E_{1, i, j}^{t}-x_{1, i}^{t+1}}{\delta z_{l}}-Q_{x, i}^{t+1}\right) .
$$

Furthermore, the surface energy balance for each tile can be written as

$$
C_{s, i} c_{p}^{-1} \frac{\partial s_{\text {surf }, i}}{\partial t}=R_{n, i}+G_{i}+L E_{i}^{*}+H_{i}^{*} .
$$

Together with the equations for the surface heat and moisture fluxes below the lowest level of the atmosphere (Eq. 16), three times the number of tiles equations are obtained that can 
be solved for the three times the number of tiles unknowns $s_{1, i}^{t+1}, q_{1, i}^{t+1}$ and $s_{\text {surf }, i}^{t+1}$ (for further details see supplementary material).

\section{Single-Column Model Studies}

Single-column experiments are especially well suited to investigate processes and mechanisms related to the land-surface-atmosphere coupling. On one hand, they incorporate substantially less degrees of freedom than global scale GCMs, and the absence of large-scale atmospheric effects makes results much easier to interpret. On the other hand, the entire vertical column including all relevant processes is simulated, which allows the investigation of feedback effects between surface and atmosphere.

A single-column model can be considered as a very primitive model of the atmosphere and the surface. It reproduces the processes within a representative model grid box rather than those given at a specific location in the real world. Therefore, the following investigations do not attempt to prove that a given coupling scheme gives better results with respect to reality, but that the choice of coupling scheme may have a substantial impact on the simulated state of a grid box. In single-column simulations, the prescription of an advective forcing is required in order to account for the atmospheric meridional heat and moisture transport from equatorial to polar regions. Disregarding the advective forcing leads to an excess in energy in equatorial regions and an energy deficit in high latitudes that leads to extreme and unrealistic states simulated in the respective regions. However, in extreme scenarios certain interdependencies, e.g., surface temperature and soil moisture, may become more clear. Thus, it may even be more instructive to investigate processes in the extreme scenarios given when an advective forcing is omitted.

Results are presented below using the one-dimensional version of the Max Planck Institute for Meteorology's Earth-system model (Raddatz et al. 2007; Brovkin et al. 2009; Stevens et al. 2013), in which the VERTEX scheme described in Sect. 2 has been included. The model comprises the atmospheric model ECHAM6 and the land-surface model JSBACH . The respective simulations were performed with the model standard resolution, i.e., a vertical resolution of 47 model levels, with the lowest three levels located at heights of roughly 30,150 and $350 \mathrm{~m}$, a horizontal resolution of T63, which corresponds to a grid spacing of about $200 \mathrm{~km}$ and a temporal resolution of $20 \mathrm{~min}$. In the operational set-up, the atmospheric turbulent fluxes are modelled by a modified version of the turbulent kinetic energy (TKE) scheme described in Brinkop and Roeckner (1995). The scheme uses the TKE, the turbulent mixing length (Blackadar 1962) and a stability function that depends on the moist Richardson number (Mellor and Yamada 1982) to describe the turbulent viscosity and diffusivity. In the scheme, the dissipation rate is dependant on the dissipation length scale, which is assumed to be equal to the mixing length. For convectivly unstable situations, the bottom boundary condition is determined by the stability of the surface layer and depends on the friction velocity, the convective velocity scale and the Obukhov length (Mailhot and Benoit 1982). In the VERTEX scheme, the formulations are used in essentially the same way, but calculations are performed for the individual tiles separately within the lowest three layers of the atmosphere.

The standard model version uses a parameter-aggregation scheme in which the aggregation to the effective grid-box parameters is performed according to Kabat et al. (1997) and Lemmel and Helenius (1998), more specifically, the aggregation of the roughness length follows Mason (1988), Claussen (1991) and Claussen et al. (1994) and the aggregation of the albedo follows Otto et al. (2011). Based on the effective parameters, the surface fluxes are calculated 
using a bulk-exchange formulation that applies approximate analytical expressions similar to those proposed by Louis (1979) to determine the transfer coefficients. Evapotranspiration is given by (Schulz et al. 1998),

$$
E=c_{\mathrm{cp}} \beta\left(q_{1}-\alpha q_{\mathrm{sat}}\left(c_{p} s_{\mathrm{surf}}\right)\right) .
$$

For potential evaporation, without limitations due to the availability of water, and snow sublimation, the coefficients are $\beta=\alpha=1$, and whenever the soil is not fully saturated, bare soil evaporation is reduced according to Roeckner et al. (1996). For transpiration, $\alpha=1$, and $\beta$ is calculated based on Sellers et al. (1986). In the VERTEX scheme, the surface fluxes are calculated accordingly, but for individual tiles and not for the entire grid box. In the soil, a horizontal flow of water and heat is not represented in the model, and the soil moisture and temperature in a given tile is independent of the other tiles.

The VERTEX scheme has been implemented in such a way that the atmospheric processes included in the model can be divided into grid-resolved and tile-resolved processes. The airmass properties, i.e., temperature and specific humidity, of the lowest model levels are resolved with respect to the surface tiles, but these tile specific characteristics are being considered only in the process of turbulent mixing. To maintain the level of complexity at a minimum, SGS heterogeneity of the wind field was not taken into account in the computation of TKE. All other processes such as convection, radiative heating and precipitation are gridresolved processes, thus they exclusively depend on grid mean values.

A simple approach was followed, to indirectly account for mesoscale circulations. They were taken into account in the calculation of the blending height rather than by a designated factor in the calculation of $d_{z, i}$ and $m_{z, i, j}$. It was assumed that mesoscale circulations occur exclusively due to differential surface heating during unstable conditions. In order to increase $m_{z, i, j}$ accordingly, the blending heights were calculated based on the more general formulation of the blending height (Eq. 1 with $C=1, p=2$ ), even though buoyancy-driven turbulence (Eq. 2) results in much larger blending heights under these circumstances. It has to be acknowledged that, if the balancing effects of mesoscale circulations are small, this simplification leads to an underestimation of the blending height. $d_{z, i}$ was calculated as the ratio of height above ground $h_{z}$ and blending height $h_{\mathrm{blend}, i}$, and $m_{z, i, j}$ was calculated based on Eq.4,

$$
\begin{aligned}
h_{\text {blend }, i} & =\left(\frac{u_{*, i}}{U}\right)^{2} L_{\text {hetero }, i}, \\
d_{z, i} & =\min \left(1, \frac{h_{z}}{h_{\text {blend }, i}}\right) .
\end{aligned}
$$

In the present model set-up, the resolution of air properties has been limited to the lowest two atmospheric model levels in order to limit computational expenses, even though the calculated blending height may lay much above the third lowest level. Therefore, on the lowest (around $30 \mathrm{~m}$ ) and second lowest model level (around $150 \mathrm{~m}$ ), the extent to which fluxes have horizontally blended linearly depends upon the blending height, whereas on the third lowest level (around $350 \mathrm{~m}$ ), fluxes are assumed to be completely horizontally homogeneous. In the present study, SGS heterogeneity is constituted by clusters of different land-cover types that can be found within a given model grid box. To approximate the characteristic length scales $L_{\text {hetero, } i}$ of these clusters at the model resolution ( $T$ 63), the GlobCover dataset (Arino et al. 2012) was used. An exception to this are the characteristic length scales in the first study, which were not derived from the dataset but chosen arbitrarily. The horizontal wind speed $U$ at the blending height and the friction velocity $u_{*, i}$ are calculated within the model. 


\subsection{Wet vs. Dry Tile}

The first simulation is performed for an idealized grid box located at $23.3^{\circ} \mathrm{N}, 75.0^{\circ} \mathrm{E}$, which comprises a wet and a dry tile. Half of the grid box is assumed to be covered by irrigated crops (wet tile) and the other half by rainfed crops (dry tile), both of which exist in clusters with a characteristic length scale of $50 \mathrm{~km}$. The vegetation characteristics of both crops are identical, and the two tiles only differ with respect to the treatment of soil moisture. At the beginning of each timestep, the soil moisture content within the wet tile is set to the value at which potential transpiration is reached, i.e., $75 \%$ of the water holding capacity $(0.53 \mathrm{~m})$. In contrast to this, the dry tile is initialized with the soil moisture content at the wilting point $(0.21 \mathrm{~m})$, i.e the minimum soil moisture required for plants not to wilt. The simulation was performed for the months of July and August 1992, using ERA-Interim reanalysis data for initialization (Dee et al. 2011) (for more details on the initial conditions see supplementary material). In the following, a period from the 16 to 18 July is used to demonstrate characteristic aspects of the new coupling scheme. Before and during this period no rainfall occurs, and there is no drainage or evapotranspiration within the dry tile (as the soil moisture is at the wilting point and there is no water present in the upper $0.1 \mathrm{~m}$ of the soil column from which it can evaporate) so that the soil moisture values of both tiles are identical to the initialization values.

In Sect. 2 the numeration of model levels starts with the lowest level in the atmosphere as level 1, and the numbers increase until the top of the atmosphere. Consequently, in the following sections, the lowest atmospheric level is referred to as $l_{1}$ (located at a height of $\approx 30 \mathrm{~m})$, the second lowest and third lowest levels as $l_{2}(\approx 150 \mathrm{~m})$ and $l_{3}(\approx 350 \mathrm{~m})$.

\subsubsection{Surface Fluxes and Blending Heights}

In the JSBACH model the magnitude of the turbulent surface flux of a given variable (see Fig. 3c) depends on the respective surface atmosphere gradient as well as on the product of the horizontal wind speed and the surface drag coefficient. In the following, this product is referred to as the coupling coefficient $c_{\mathrm{cp}}$. The surface drag coefficient depends on the roughness length and the stability between surface and lowest atmospheric level. As the horizontal wind speed is calculated for the entire grid box and the roughness length is similar in both tiles, differences in $c_{\mathrm{cp}, i}$ can be used as a proxy for stability differences.

The coefficient associated with the dry tile is much larger than that in the wet tile (see Fig. 3b). The reason for this are higher surface temperatures within the dry tile (see Fig. 3a), which result in a less stable stratification of the lowest layers of the atmosphere. For most of the 3-day period $c_{\mathrm{cp} \text {, dry }}$ is more than two thirds larger, and in the periods of surface cooling in the afternoon, $c_{\mathrm{cp} \text {, dry }}$ may even be orders of magnitude larger than $c_{\mathrm{cp} \text {, wet }}$. This means that the exchange of air properties between the surface and the atmosphere is distinctly more pronounced within the dry tile.

Figure 4 shows the potential blending heights calculated for each tile using Eq. 1. Due to the large horizontal extents of the crop clusters, the blending height is much higher than the lowest three atmospheric model levels. The blending height attains its maximum around noon, with values between 1500 and $2500 \mathrm{~m}$ in the wet tile and between 3000 and $4500 \mathrm{~m}$ in the dry tile. This implies that, in theory, the vertical fluxes do not become horizontally homogeneous within the PBL for a large part of the day.

For the horizontal length scales of the crop tiles, the blending height cannot be used to predict the height above which in reality the state of the atmosphere becomes horizontally homogeneous. The concepts on which it is based do not extend past the PBL into the free 


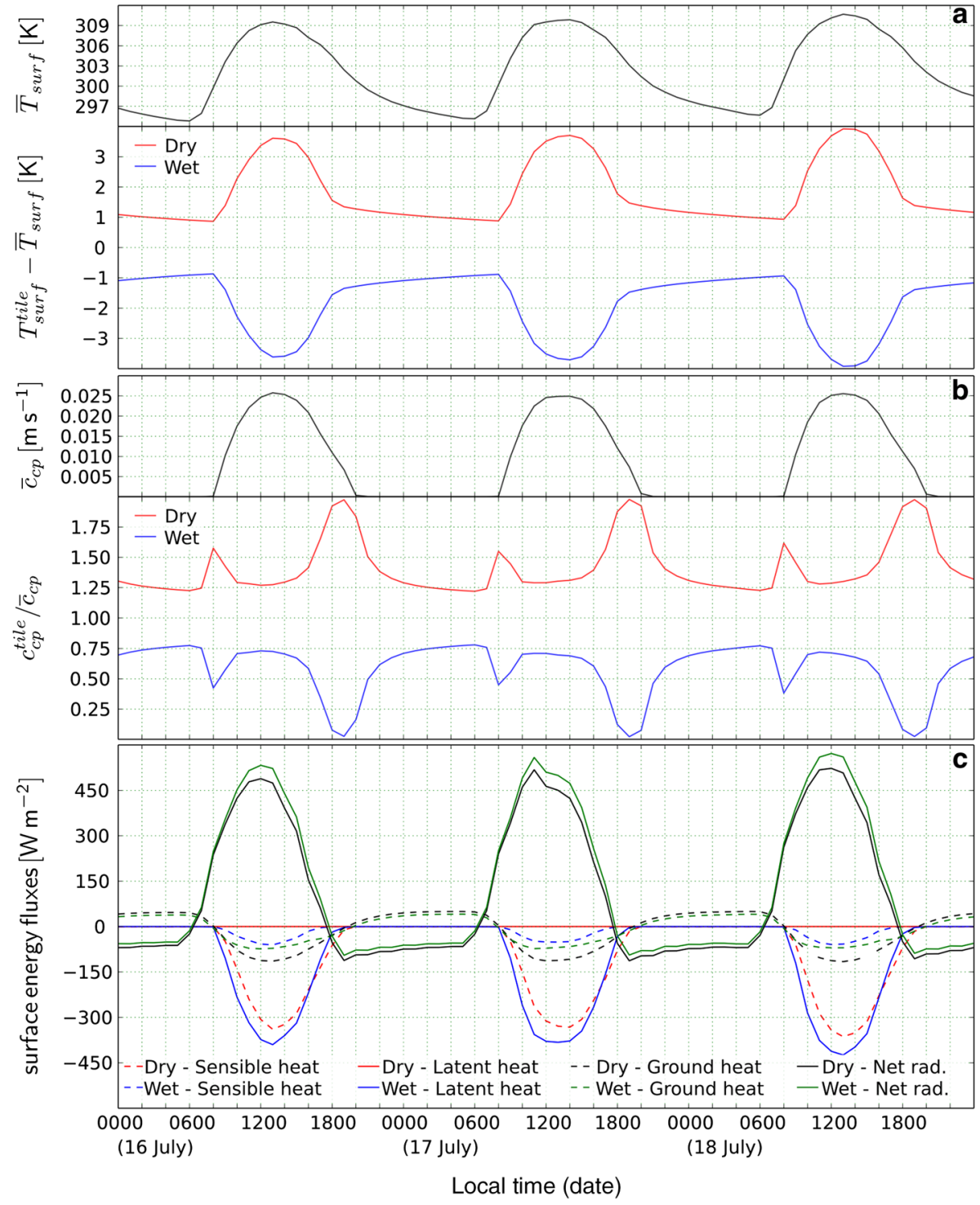

Fig. 3 a Grid-box mean surface temperature (top) and tile specific deviations from the mean (bottom), b grid-box mean surface coupling coefficient (top) and tile specific coupling coefficients relative to the mean (bottom), c surface energy fluxes; $23.3^{\circ} \mathrm{N}, 75.0^{\circ} \mathrm{E}$

atmosphere. Consequently, for these length scales, the potential blending height merely represents the height at which the atmospheric state would become horizontally homogeneous if it was located below the boundary-layer top. However, it is still conceivable to use the potential blending height as a scaling factor for the horizontal blending of the vertical fluxes within the PBL. There is evidence that, even for length scales $L_{\text {hetero, } i}<<50 \mathrm{~km}$, the atmosphere is not necessarily horizontally homogeneous at heights of more than $1 \mathrm{~km}$ and that heterogeneity extends to the top of the CBL when $L_{\text {hetero, } i}$ becomes significantly larger than the 


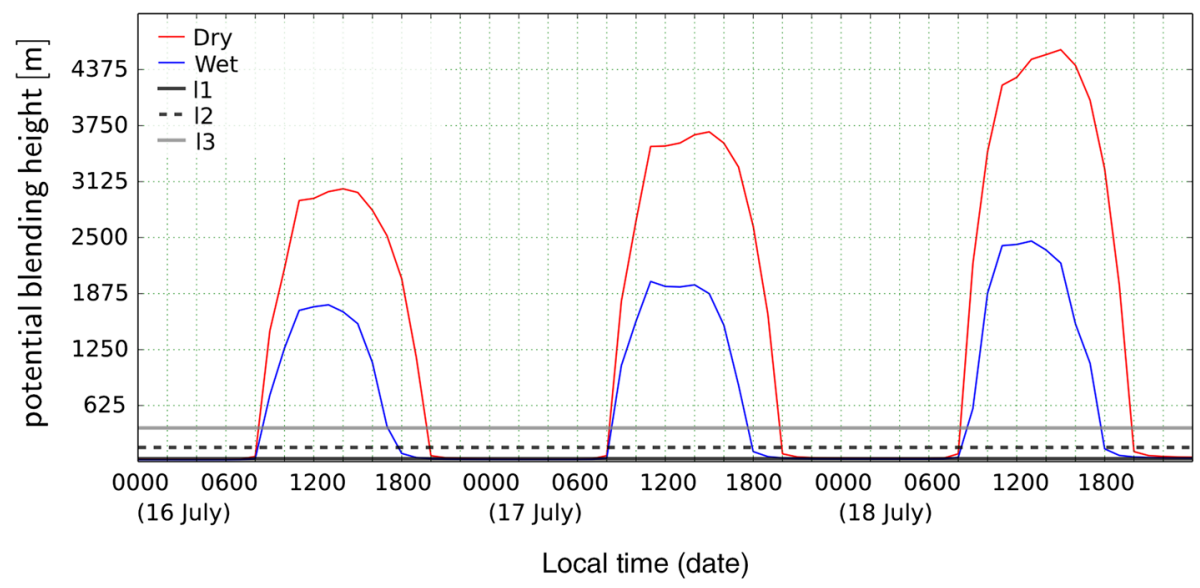

Fig. 4 Potential blending height and heights of the lowest model levels; $23.3^{\circ} \mathrm{N}, 75.0^{\circ} \mathrm{E}$

height of the CBL (Strunin et al. 2004). Here, the Raupach length $L_{\mathrm{RAU}}$, as a function of the Deardorff velocity scale, the height of the CBL and the wind speed, may be used as a horizontal length scale of surface features whose influence is confined to the CBL (Raupach and Finnigan 1995). When assuming that the heterogeneity at the top of the PBL depends linearly on the ratio of $L_{\mathrm{RAU}}$ and $L_{\mathrm{hetero}, i}, d_{z, i}$ can also be expressed as a function of $L_{\mathrm{RAU}}$ and the height of the PBL $h_{\mathrm{bl}}$,

$$
d_{z, i}=\frac{L_{\mathrm{RAU}}}{L_{\mathrm{hetero}, i}} \frac{h_{z}}{h_{\mathrm{bl}}} .
$$

However, there is no conclusive evidence that this approach provides a more accurate description of the actual extent to which the vertical fluxes blend horizontally with height. As $d_{z, i}$ is predominantly smaller for Eq. 20 than for Eq. 19, $d_{z, i}$ was calculated based on the potential blending height with the aim of rather underestimating the effect of heterogeneity than to overestimate it.

\subsubsection{Atmospheric Temperature and Specific Humidity}

Using the blending height as a scaling factor, between roughly 0900 local time (LT) and $1700 \mathrm{LT}$, the extent to which fluxes have horizontally blended at level $l_{1}$ is below $3 \%$ and at level $l_{2}$ below $15 \%$. Thus, it is remarkable that the temperature deviations at the levels $l_{1}$ and $l_{2}$ have been reduced by an order of magnitude in comparison to the deviations at the surface (Figs. 3a, 5a). The reason for this strong decline is a stability-related mechanism that, in predominantly unstable situations, equilibrates the temperatures in the tiles. Assuming that the properties of air become horizontally more homogeneous with height, the stability in a tile decreases relative to the other tiles when the air in this tile becomes warmer than in the others. This causes an increase in vertical turbulent mixing relative to the colder tiles. Because the air at the level above is colder, this relative increase in turbulent transport results in a relative increase in the mixing with colder air within the warmer tile, thus equilibrating temperatures. Therefore, the reduction of tile specific deviations with height does not scale linearly with the extent to which fluxes have become horizontally homogeneous. In the simulation, this effect can be seen in the differences in eddy diffusivity between tiles. Between roughly 0800 LT 


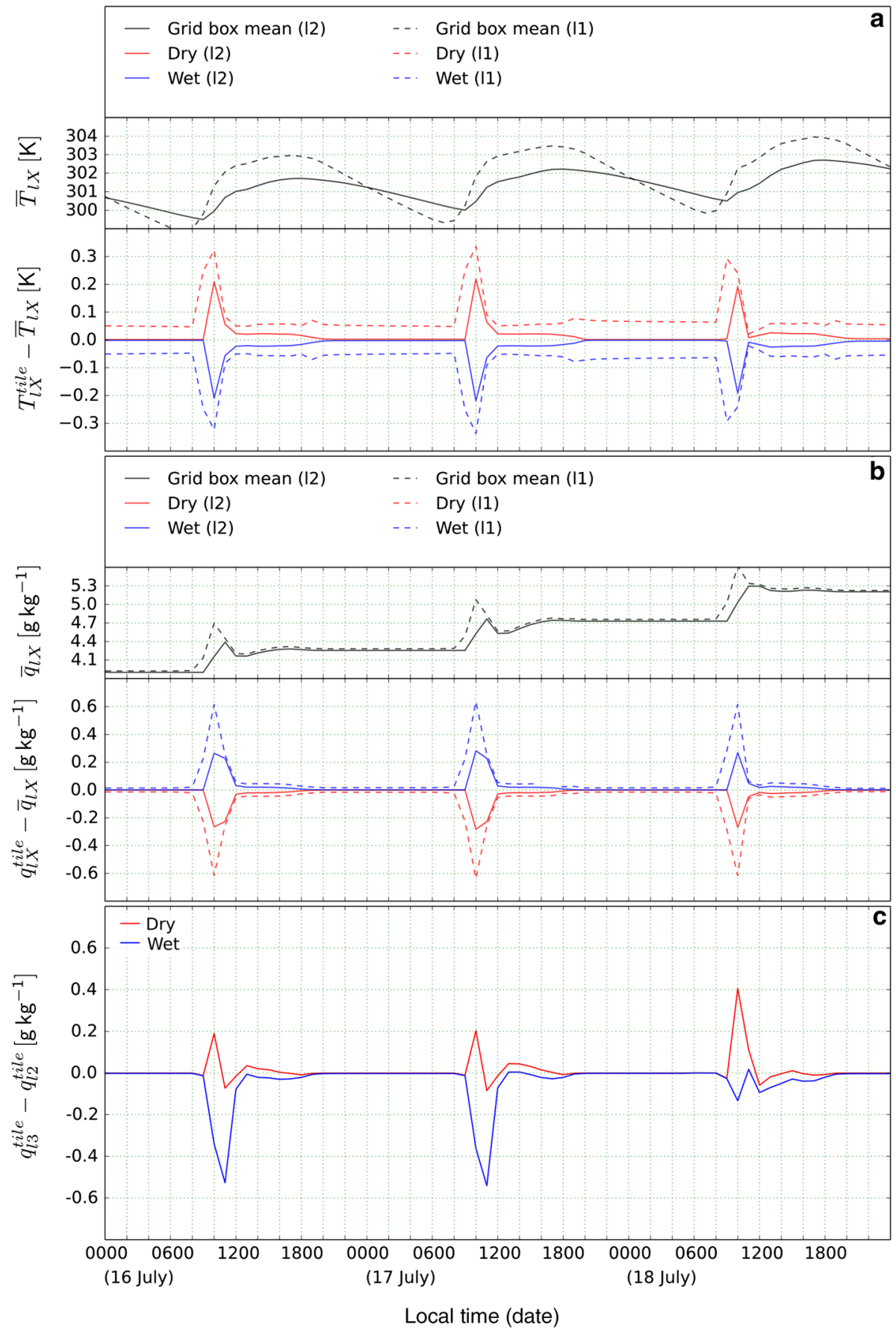

Fig. 5 a Grid-box mean temperatures at levels $l_{2}$ and $l_{1}$ (top) and the respective tile specific temperature deviations (bottom), b grid-box mean specific humidity at levels $l_{2}$ and $l_{1}$ (top) and the respective tile specific humidity deviations (bottom), c specific humidity difference between levels $l_{3}$ and $l_{2} ; 23.3^{\circ} \mathrm{N}, 75.0^{\circ} \mathrm{E}$ 


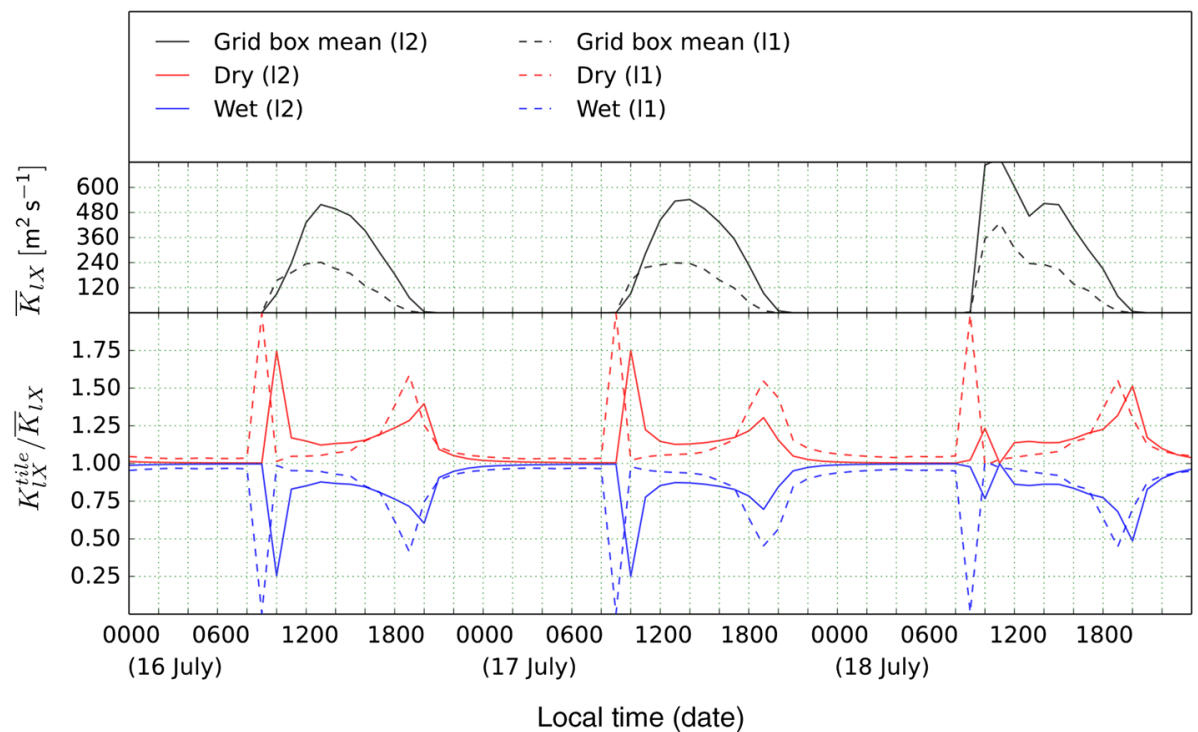

Fig. 6 Grid-box mean eddy diffusivity between levels $l_{2}$ and $l_{3}$ and between levels $l_{1}$ and $l_{2}$ (top) and the respective tile specific eddy diffusivities relative to the mean (bottom), (the eddy diffusivity between levels $l_{1}$ and $l_{2}$ is indexed by the lower level $l_{1}$ and that between levels $l_{2}$ and $l_{3}$ is indexed by level $l_{2}$ ); $23.3^{\circ} \mathrm{N}, 75.0^{\circ} \mathrm{E}$

(0900 LT at level $l_{2}$ ) and $2100 \mathrm{LT}$, the eddy diffusivity between two levels is on average more than $50 \%$ larger in the dry tile than in the wet tile (Fig. 6).

The top panel of Fig. 5b shows that the atmospheric specific humidity is not in an equilibrium state. Due to the increasing temperatures and the abundance of moisture at the surface within the wet tile, the grid mean specific humidity at the two lowest atmospheric levels increases throughout the 3 -day period by more than $1 \mathrm{~g} \mathrm{~kg}^{-1}$. Between $0800 \mathrm{LT}$ and $1000 \mathrm{LT}$, the tile specific deviations in specific humidity are large compared to the grid mean with a peak of about $0.6 \mathrm{~g} \mathrm{~kg}^{-1}$ at level $l_{1}$ and about $0.25 \mathrm{~g} \mathrm{~kg}^{-1}$ at level $l_{2}$ (see Fig. $5 \mathrm{~b}$ ). This shows that the increase in atmospheric humidity occurs to a large extent within the wet tile, whereas the specific humidity in the dry tile increases only slightly, which agrees with the small extent to which fluxes have blended horizontally. Consequently, when the mixed layer grows and the properties of the relatively humid air, which is largely located above the wet tile, are being mixed with the properties of the dryer more homogeneous air from above, the SGS humidity variability is strongly reduced.

Because the states of the individual tiles converge with increasing height, the vertical moisture gradient is smaller in the dry tile than in the wet tile. Therefore, the upward moisture flux within the dry tile is very small when compared to the wet tile, and the moisture, which is mixed into the air above the dry tile from the wet tile, largely remains within the lowest layers of the atmosphere. For certain periods, the moisture fluxes within the tiles even have opposing directions so that a process develops that to some extent resembles a mesoscale circulation. In the dry tile between roughly 0900 LT-1100 LT and 1200-1600 LT, the specific humidity at level $l_{3}$ (here fluxes have become horizontally homogeneous) is larger than at level $l_{2}$ (see Fig. 5c). This means that during these periods moisture is being mixed upwards above the blending height within the wet tile and transported downwards within the dry tile, thus equilibrating the differences in specific humidity between the tiles. Therefore, for the longest part of any day, the SGS humidity variability is very small, despite the fact that no evapotranspiration occurs in the dry tile and fluxes have horizontally blended by less than 
$3 \%$ at level $l_{1}$ and less than $15 \%$ at level $l_{2}$. This is in accordance with studies that indicate that fluxes are blended at greater heights than scalars (Brunsell et al. 2011).

\subsection{Temperature Inversion}

The second idealized study was performed for a grid box located at $64.4^{\circ} \mathrm{N}, 114.4^{\circ} \mathrm{W}$, which comprises evergreen trees (about $57 \%$ ), deciduous trees (24\%) and C3 grass (19\%). The respective average horizontal extents at the surface are about $2000 \mathrm{~m}$ (evergreen trees), $1700 \mathrm{~m}$ (deciduous trees) and $3000 \mathrm{~m}$ (C3 grass). The simulation was performed for the months of January and February 1992, and in the following, a period from 1 to 5 January will be used to demonstrate characteristic aspects of the new coupling scheme for the case of a temperature inversion in the lowest layers of the atmosphere. A spin-up phase is not taken into consideration, as some of the most interesting aspects related to the new coupling scheme are most eminent before an equilibrium state is reached. Furthermore, the height of the stable PBL that forms is mostly below $150 \mathrm{~m}$. In this case, due to the GCM's low vertical resolution, the entire PBL is represented by merely one model level. This makes the formulation of important boundary-layer processes problematic, so that the case should be taken as a strongly idealized study rather than an exact representation of reality.

It is notable that during the simulation the tile specific temperature deviations at level $l_{1}$ become larger than those at the surface (see Fig. 7a). This is striking, as the blending height during the experiment was very low (below level $l_{2}$ ) and the coupling scheme is set-up in such a way that the vertical fluxes become horizontally more homogeneous with height. Furthermore, the local stratifications are such that relatively warmer air is located above the relatively colder surface and vice versa. Intuitively one would expect that a convergence of fluxes with increasing height would necessarily result in a convergence of the states over the different tiles. However, the underlying process is to a certain degree self enhancing, allowing the initially small deviations in the atmosphere to grow larger than those at the surface. On level $l_{1}$ the air in the grass tile is initially slightly warmer than in the tree tiles. Within the warmer tile, the temperature deviations in the atmosphere facilitate a relative decrease in stability between the two lowest levels of the atmosphere and a relative decoupling from the surface (indicated by the differences in $c_{\mathrm{cp}}$ and eddy diffusivity in Fig. 7b, c). Consequent there is a relative increase in the exchange with the warmer air from above the blending height and a decrease in the mixing with the cold near-surface air in the warmer tile. This, in turn, promotes the increase in temperature deviations at level $l_{1}$ and thus, an increase in stability differences. These again have a positive feedback on the downward mixing of heat towards level $l_{1}$ and a negative feedback on the downward mixing of heat towards the surface in the warmer grass tile. This process is only interrupted when surface temperatures increase, due to an increase in incoming shortwave radiation (not shown here), and consequently, the stability between surface and level $l_{1}$ decreases.

The air at level $l_{2}$ interacts only with the grid-box average flux to which the different tiles contribute according to their share in grid-box area. The grass tile has a comparatively low share in grid-box area $(19 \%)$. Thus, the relative temperature increase at level $l_{1}$ in the grass tile and the temperature decrease at level $l_{2}$, resulting from the vertical turbulent mixing between the two levels, are disproportional. This disproportionality prevents the temperature at level $l_{2}$ from rapidly approximating to the temperature of the grass tile at level $l_{1}$, which would limit the downward transport of heat. In this constellation, the horizontal convergence of fluxes with height does not result in a convergence of the states of the different tiles with increasing height, but actually facilitates their divergence. Furthermore, it enables a 


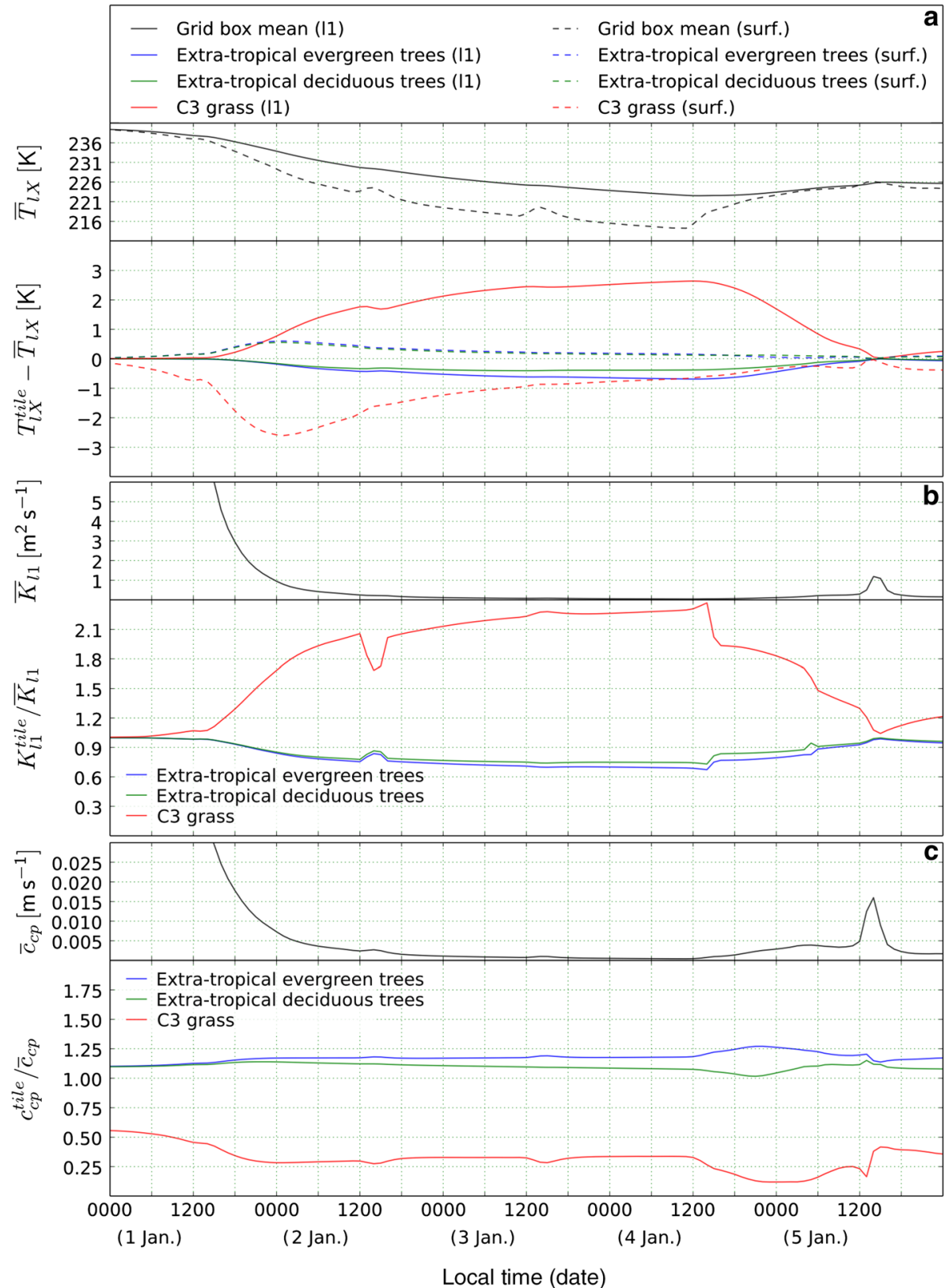

Fig. 7 a Grid-box mean temperature at level $l_{1}$ and at the surface (top) and the respective tile specific temperature deviations (bottom), b grid-box mean eddy diffusivity between levels $l_{1}$ and $l_{2}$ (top) and the respective tile specific eddy diffusivities relative to the mean (bottom), c grid-box mean surface coupling coefficient (top) and the respective tile specific coupling coefficients relative to the mean (bottom); $64.4^{\circ} \mathrm{N}$, $114.4^{\circ} \mathrm{W}$

constellation in which relatively colder air is located above a relatively warmer surface and vice versa. 
In this magnitude, the process described above is not a generally observable feature of the VERTEX scheme, as it was only found under the specific circumstances described at the beginning of this section. Furthermore, it is questionable whether atmospheric spatial variability that exceeds the causative variability at the surface can be observed in the real world. Therefore, the above example should merely be understood as an emphasis on the strong nonlinearity in the processes involved. Nonetheless, small-scale numerical studies indicate that local stratifications are plausible in which areas of warmer air located at a certain height above smoother and colder surfaces adjoin rougher and warmer surfaces on top of which cooler air is located, resulting in distinct atmospheric spatial heterogeneity (Mott et al. 2015). Generally, the underlying mechanism of local decoupling is not well understood, but could represent an important factor in e.g., snow patch survival (Derbyshire 1999; Mott et al. 2013).

\subsection{Comparison of Different Coupling Schemes}

The above simulations were analyzed with respect to the behaviour of the turbulent vertical transport when the lowest atmospheric model levels are further partitioned into the same tiles as the surface. However, the resolved transport process has not yet been compared to the vertical turbulent transport under the assumption of the blending height below the lowest model level.

For this comparison, simulations of 35 grid boxes using the parameter-aggregation (PARAM), the simple flux-aggregation (SIMPLE-FLUX) and the VERTEX scheme (VERTEX) were performed for the duration of one year. The grid boxes, which cover different longitudes, latitudes and climate zones, were selected in such a way as to include tiles with diverse characteristics, large cover fractions and horizontal extents.

In the framework of a single-column simulation, the differences between two simulations can become unrealistically large because of the absence of horizontally compensating processes. For example, if in a grid box all soil moisture is evaporated (hence all incoming radiation is expended in surface warming and the sensible heat flux) a drastic temperature increase cannot be compensated by horizontal heat or moisture advection. If this is the case in only one of the simulations, the difference to the other simulations can be dramatic. Therefore, the subsequent results present idealized cases, and the differences in the state of individual grid boxes are potentially much larger than they would be in a global simulation.

It can immediately be seen that the choice of the coupling scheme has a substantial impact on the annual mean state of the simulated grid boxes (Fig. 8). Temperature differences may exceed $20 \mathrm{~K}$, differences in specific humidity $10 \mathrm{~g} \mathrm{~kg}^{-1}$, and differences in cloud cover may be larger than $50 \%$ in certain grid boxes and levels. In comparison to the parameter-aggregation scheme, the two flux-aggregation schemes exhibit a clear tendency to produce a colder and dryer atmosphere, with more clouds lower and less clouds higher up in the atmosphere. Even though this is not true for all grid boxes, on average the temperature differences between the SIMPLE-FLUX simulations (VERTEX simulations) and the PARAM simulations slightly exceed $-3 \mathrm{~K}(-0.75 \mathrm{~K})$ between the surface and the 22nd model level (around $14 \mathrm{~km})$. On average, the differences in specific humidity become as large as $-0.8 \mathrm{~g} \mathrm{~kg}^{-1}\left(-1.6 \mathrm{~g} \mathrm{~kg}^{-1}\right)$ close to the surface and about $-1.3 \mathrm{~g} \mathrm{~kg}^{-1}\left(-0.8 \mathrm{~g} \mathrm{~kg}^{-1}\right)$ between level 8 and 15 (between 2.5 and $7 \mathrm{~km})$.

As shown above, different tiles have different strength in coupling between the atmosphere and the surface. As this is dependent on the amount of turbulence created, tiles with a large roughness length have a stronger coupling between surface and atmosphere. In the JSBACH model the tiles that represent plant functional types with a large roughness length such as trees are also those with a pronounced ability to transpire. The concurrence of large roughness 

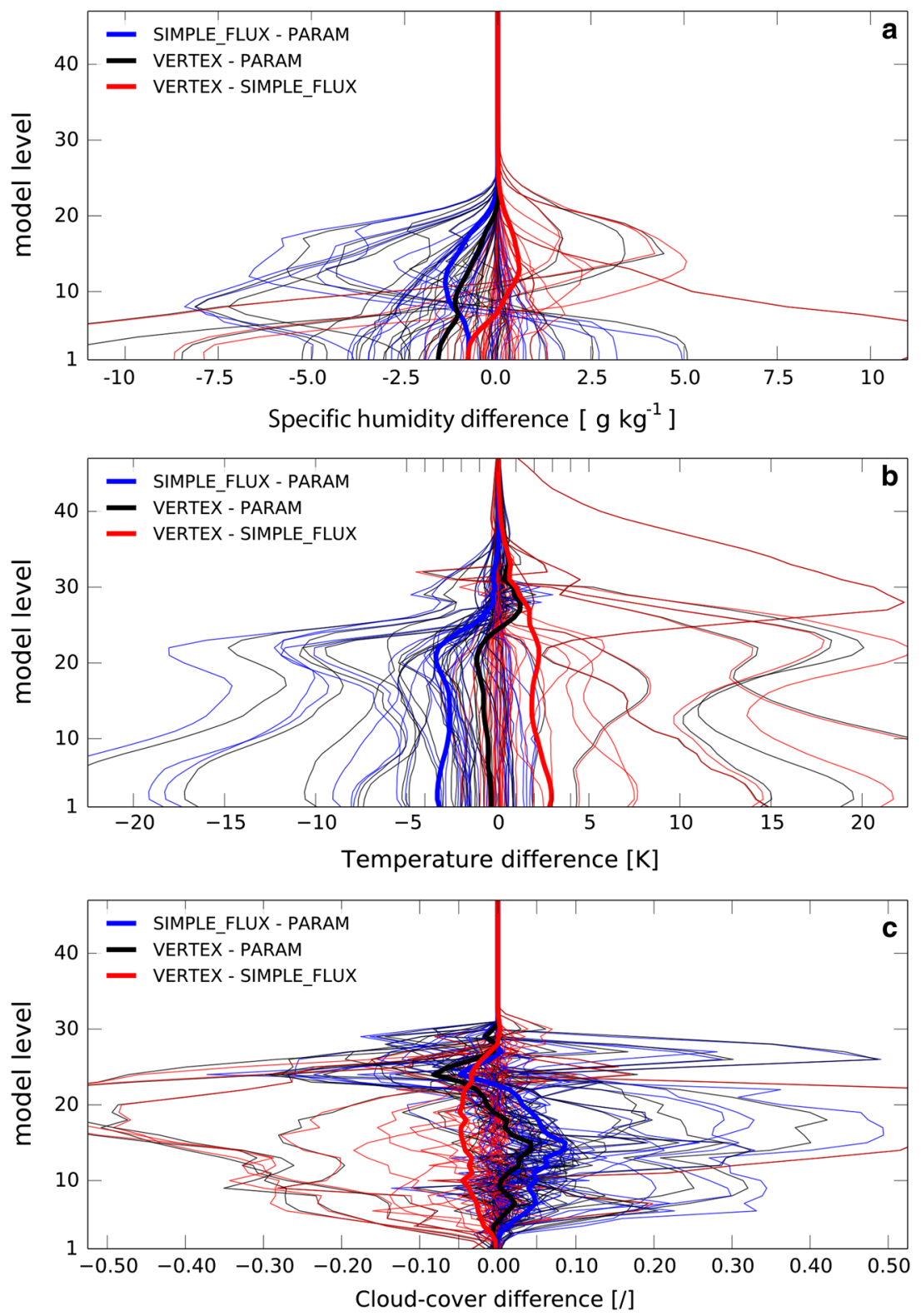

Fig. 8 Differences in annual mean of 35 simulated grid boxes a specific humidity, b temperature, $\mathbf{c}$ cloud cover, thin lines correspond to the individual grid boxes and the thick line to the average of all boxes

lengths and transpiration rates above the grid mean promotes the upward transport of moisture from the surface. This effect is further intensified by the fact that the albedo of these tiles is predominantly below the grid mean, which means that also more energy from absorbed shortwave radiation is available. Thus, by resolving the surface fluxes with respect to the tiles, there is an initial increase in grid mean upward latent and decrease in upward sensible heat 
flux. This shift in Bowen ratio initially leads to a colder and moister state of the atmosphere when using a flux-aggregation scheme.

The difference in the atmospheric state has a strong effect on the formation of clouds, as it leads to an increase in cloud cover lower in the atmosphere, and consequently on the radiation budget at the surface. Furthermore, the colder and moister atmosphere initially facilitates the occurrence of precipitation. Precipitation is a process that is not resolved with respect to the tiles. Hence, all tiles receive the same amount of precipitation relative to their cover fraction. At the same time, the proportionally larger share of the atmospheric water vapour, which precipitates, originates from tiles with pronounced transpirational abilities and roughness lengths. This eventually leads to an accumulation of soil moisture within the tiles with poor transpirational abilities and possible water stress in tiles with pronounced transpirational abilities. With the large share of water being located in the tiles that transpire at rates below the grid mean, on average there is a strong decrease in the upward latent heat flux and the vertically-integrated water vapour is reduced. Due to the reduction in incoming radiation, less energy is available at the surface and, not only the latent heat flux decreases but also the upward sensible heat flux. Thus, in the larger share of the simulated grid boxes, temperatures and specific humidity rates are lower when a flux-aggregation scheme was used for the simulation.

The effects described above are more pronounced for the SIMPLE-FLUX simulations than for the VERTEX simulations. Thus, there are also large differences between the simulations with the two flux-aggregation schemes. In the VERTEX simulations, there is a distinct tendency towards higher temperatures between the surface and the 27th model level (around $21 \mathrm{~km}$ ), smaller specific humidity values close to the surface and larger specific humidity values further up in the atmosphere [between levels 7 and 22 (between 2 and $14 \mathrm{~km}$ )]. The simulations performed with the VERTEX scheme gave an atmospheric state that is on average between 2 and $3 \mathrm{~K}$ warmer than in the SIMPLE-FLUX simulations. Close to the surface the atmosphere is about $0.8 \mathrm{~g} \mathrm{~kg}^{-1}$ dryer and moister higher up.

The reason for this lies within the resolution of the vertical transport process in the lowest layers of the atmosphere with respect to the tiles. As has been argued, the atmospheric column within the warmer tiles is predominantly less stable than in the colder tiles, which facilitates the vertical mixing. A stronger vertical exchange within the relatively warmer and dryer tiles means that, in the VERTEX simulations, initially more sensible heat relative to moisture is being transported upwards from the surface in comparison to the SIMPLE-FLUX simulations. At the same time, more moisture remains in the lowest layers of the atmosphere, relatively reducing the atmospheric moisture demand. The consequent shift from surface latent to surface sensible heat flux results in an initially warmer and dryer atmosphere.

Due to the temperature increase in the atmosphere, the saturation vapour pressure increases. This feeds back on the formation of clouds, and below the 28th model level (around $22 \mathrm{~km}$ ) the cloud cover is strongly reduced. Consequently, less moisture is removed in the form of precipitation, leading to an overall increase in specific humidity higher up in the atmosphere and an increase in vertically-integrated water vapour. Furthermore, the reduction in cloud cover leads to a strong increase in net shortwave radiation. The reduction in precipitation limits the availability of water at the surface and strongly reduces annual evapotranspiration. This, together with the increase in available energy, due to the increase in net radiation, causes an increase in surface and atmospheric temperatures and in the upward sensible heat flux.

As can be seen in Fig. 8, the response to a certain coupling scheme, as described above, is not represented by all the example grid boxes. Thus, the arguments given here pertain rather to an idealized mean scenario than to a specific simulation. This is because, even in 
a single grid box, the processes are much more complex than what has been reasoned. The initial tendency, i.e., a relative increase in evapotranspiration related to the flux-aggregation schemes and a more pronounced vertical exchange in the relatively warmer tiles when using the VERTEX scheme, could be found in almost all of the simulated grid boxes. However, the individual grid boxes exhibited strongly diverging responses to this initial tendency. For example, a warmer surface may lead to a decrease in stability and a more frequent triggering of convection and a consequent increase in (cumulus) cloud cover. Increased precipitation early in the year could lead to clearer skies and an increase in incoming shortwave radiation, resulting in a warmer atmosphere, despite an initially higher relative humidity. Water stress may affect the behaviour of plants and by this the surface albedo, which in turn affects the energy balance at the surface. Nonetheless, as the initial tendency related to a certain coupling scheme is systematic, the simulations performed with a given coupling scheme favour a development in a certain direction.

\section{Conclusions and Discussions}

Modelling the link between the atmosphere and the horizontally heterogeneous land surface remains a key challenge for present day weather and climate models. The present study investigated the influence of surface heterogeneity on the turbulent mixing process, using the newly developed VERTEX scheme. By taking into account horizontal heterogeneity, not only at the surface, but also at the lowest levels of the atmosphere, the scheme allows the resolution of the turbulent mixing process with respect to the surface tiles. Here, it could be shown that the intensity of the vertical turbulent mixing process can differ largely within a grid box. It has been argued that these differences are closely connected to the differences in atmospheric stability between the different tiles, which can only be taken into account by resolving the lowest layers of the atmosphere with respect to the surface tiles.

Two cases have been used to demonstrate how differences in atmospheric stability relate to the tile specific deviations of the atmospheric state and of the state of the surface. It could be shown that the SGS variability in the state of the atmosphere does not necessarily scale linearly with height. In comparably unstable circumstances, the differences in stability help to equilibrate the state of the individual tiles, and the SGS variability in temperature and humidity decreases strongly with increasing height. Here, the a VERTEX scheme was capable of representing SGS moisture circulation, i.e., the upwards transport of moisture above the blending height in one tile and the downward transport of moisture in another tile. In contrast to this, in comparatively stable circumstances, the stability differences facilitate a divergence of the states. In extreme circumstances, this can even lead to a situation in which the differences between the state of individual tiles become larger within the lowest layers of the atmosphere than on the surface.

Furthermore, simulations performed using the VERTEX scheme were compared to simulations using a parameter-aggregation scheme and a simple flux-aggregation scheme, which does not account for horizontal heterogeneity within the atmosphere. It could be shown that, due to the non-linear nature of processes involved, the representation of SGS variability also has a distinct impact on the grid-box mean state. Here, the impact of representing SGS variability in the atmosphere is roughly half as large as the impact of an explicit representation of heterogeneity at the surface. This implies that the assumption of a blending height at the lowest atmospheric level, on which the simple flux-aggregation is based, may be the source of errors that in some cases may be as large as the errors related to the aggregation 
of parameters. Thus, employing a scheme that takes into account SGS variability within the atmosphere could significantly improve model results on the global scale.

In many of the grid boxes considered in Sect. 3.3, the impact of representing SGS variability in the atmosphere as well as at the surface is as large as the impact of an explicit representation of surface heterogeneity. Here, the system's response to a certain coupling scheme was not represented by all the selected example grid boxes. However, the system's reaction was on average systematic, so that large differences can also be expected to be found in global simulations.

Finally, in the present implementation of the VERTEX scheme, the SGS variability in the atmosphere was limited to the two lowest model levels. However, the calculated blending heights indicate that the deviations between the states of different tiles could still be large higher up in the atmosphere. As studies show, this can have a strong impact on processes such as convection and cloud formation throughout the entire PBL (Rieck et al. 2014). Here, the knowledge of SGS variability in the atmosphere, which the VERTEX scheme provides, may not only be important for its impact on the vertical turbulent transport, but it could also help to improve the representation of grid-resolved processes. Furthermore, atmospheric SGS heterogeneity of wind speed and direction was not taken into account in order to maintain the level of complexity as low as possible. As the simulations showed to be sensitive to SGS heterogeneity within the atmosphere with respect to temperature and specific humidity, this may also be the case for SGS heterogeneity with respect to wind, presenting an opportunity for future research.

For future investigations, certain constraints limit the scheme's operational applicability. Currently, it is computationally very expensive as the scheme requires solving a matrix for a number of atmospheric levels and for a number of variables. Here, the computational demand increases drastically with an increasing number of tiles that are being simulated. For a model set-up using 14 tiles, simulations (land-surface and atmospheric models) with the VERTEX scheme take almost $40 \%$ longer than simulations with the simple flux-aggregation scheme, whereas for a set-up with four tiles the computational costs are almost identical. On the one hand, this is because no optimization of the algorithms with respect to high performance machines has been undertaken, and there is a large potential for accelerating the computations, e.g., by parallelizing the matrix algorithms. On the other hand, certain structural changes in the approach may also be beneficial for simulations that require a large number of tiles. For example, a pre-aggregation of tiles based on the associated blending heights may be a useful step. Here, only the tiles above which the fluxes have not blended could be explicitly represented in the calculations on a given level, whereas the other tiles could be combined in a fraction representing the horizontally blended flux. In many present day GCMs, the PBL is not sufficiently resolved. With often less than five model levels within the lowest $1000 \mathrm{~m}$ of the atmosphere, important boundary-layer processes, especially the generation of turbulence and the development of clouds, are not represented adequately. To better capture land-surface-atmosphere interactions higher vertical resolutions close to the surface are imperative. With an increasing vertical resolution also the computational demand of the VERTEX scheme increases making an optimization even more important.

Open Access This article is distributed under the terms of the Creative Commons Attribution 4.0 International License (http://creativecommons.org/licenses/by/4.0/), which permits unrestricted use, distribution, and reproduction in any medium, provided you give appropriate credit to the original author(s) and the source, provide a link to the Creative Commons license, and indicate if changes were made. 


\section{References}

Ament F, Simmer C (2006) Improved representation of land-surface heterogeneity in a non-hydrostatic numerical weather prediction model. Boundary-Layer Meteorol 121(1):153-174

Angevine WM (2003) Urbanrural contrasts in mixing height and cloudiness over nashville in 1999. J Geophys Res 108(D3)

Arino O, Ramos Perez JJ, Kalogirou V, Bontemps S, Defourny P, Van Bogaert E (2012) Global Land Cover Map for 2009 (GlobCover 2009)

Arola A (1999) Parameterization of turbulent and mesoscale fluxes for heterogeneous surfaces. J Atmos Sci 56(4):584-598

Avissar R (1992) Conceptual aspects of a statistical-dynamical approach to represent landscape subgrid-scale heterogeneities in atmospheric models. J Geophys Res (Atmos) 97(D3):2729-2742

Avissar R, Pielke RA (1989) A parameterization of heterogeneous land surfaces for atmospheric numerical models and its impact on regional meteorology. Mon Weather Rev 117(10):2113-2136

Avissar R, Schmidt T (1998) An evaluation of the scale at which ground-surface heat flux patchiness affects the convective boundary layer using large-eddy simulations. J Atmos Sci 55(16):2666-2689

Baidya Roy S (2003) A preferred scale for landscape forced mesoscale circulations? J Geophys Res 108(D22)

Banta RM (2003) Mixing-height differences between land use types: dependence on wind speed. J Geophys Res 108(D10)

Best MJ, Beljaars A, Polcher J, Viterbo P (2004) A proposed structure for coupling tiled surfaces with the planetary boundary layer. J Hydrometeorol 5(6):1271-1278

Blackadar AK (1962) The vertical distribution of wind and turbulent exchange in a neutral atmosphere. J Geophys Res 67(8):3095-3102

Bou-Zeid E, Meneveau C, Parlange MB (2004) Large-eddy simulation of neutral atmospheric boundary layer flow over heterogeneous surfaces: blending height and effective surface roughness. Water Resour Res 40(2): $1-18$

Brinkop S, Roeckner E (1995) Sensitivity of a general circulation model to parameterizations of cloudturbulence interactions in the atmospheric boundary layer. Tellus A 47(2):197-220

Brovkin V, Raddatz T, Reick CH, Claussen M, Gayler V (2009) Global biogeophysical interactions between forest and climate. Geophys Res Lett 36(L07405):1-5

Brunsell NA, Mechem DB, Anderson MC (2011) Surface heterogeneity impacts on boundary layer dynamics via energy balance partitioning. Atmos Chem Phys 11(7):3403-3416

Claussen M (1991) Estimation of areally-averaged surface fluxes. Boundary-Layer Meteorol 54(4):387-410

Claussen M (1995) Flux aggregation at large scales: on the limits of validity of the concept of blending height. J Hydrol 166(3-4):371-382

Claussen M, Lohmann U, Roeckner E, Schulzweida U (1994) A global dataset of land surface parameters. MPI-Report 135

Cooper HJ, Smith EA, Gu J, Shewchuk S (1997) Modeling the impact of averaging on aggregation of surface fluxes over boreas. J Geophys Res (Atmos) 102(D24):29,235-29,253

Dee D, Uppala S, Simmons A, Berrisford P, Poli P, Kobayashi S, Andrae U, Balmaseda M, Balsamo G, Bechtold PBP, Beljaars A, van de Berg L, Bidlot J, Bormann N, Delsol C, Dragani R, Geera MFA, Haimberger L, Healy S, Hersbach H, Holm E, Isaksen L, Kallberg P, Koehler M, Matricardi M, McNally A, Monge-Sanz B, Morcrette J, Park B, Peubey C, de Rosnay P, Tavolato C, Thepaut J, Vitart F (2011) The era-interim reanalysis: configuration and performance of the data assimilation system. Q J R Meteorol Soc 137(656):553-597

Derbyshire S (1999) Boundary-layer decoupling over cold surfaces as a physical boundary-instability. Boundary-Layer Meteorol 90(2):297-325

Dickinson RE (1986) Biosphere/atmosphere transfer scheme (bats) for the ncar community climate model. NCAR Tech (Note TN-275+STR72)

Essery R, Best MJ, Betts RA, Taylor CM (2003) Explicit representation of subgrid heterogeneity in a gcm land surface scheme. J Hydrometeorol 4(3):530-543

Giorgi F (1997) An approach for the representation of surface heterogeneity in land surface models. Part i: theoretical framework. Mon Weather Rev 125(8):1885-1899

Giorgi F, Avissar R (1997) Representation of heterogeneity effects in earth system modeling: experience from land surface modeling. Rev Geophys 35(4):413-437

Heinemann G, Kerschgens M (2005) Comparison of methods for area-averaging surface energy fluxes over heterogeneous land surfaces using high-resolution non-hydrostatic simulations. Int J Climatol 25(3):379_ 403

Kabat P, Hutjes R, Feddes R (1997) The scaling characteristics of soil parameters: from plot scale heterogeneity to subgrid parameterization. J Hydrol 190(3-4):363-396 
Koster RD, Suarez MJ (1992) A comparative analysis of two land surface heterogeneity representations. J Clim 5(12):1379-1390

Lemmel R, Helenius N (eds) (1998) Large-scale field experiments to improve land surface parameterisations. In: Proceedings of the second international conference on climate and water

Louis JF (1979) A parametric model of vertical eddy fluxes in the atmosphere. Boundary-Layer Meteorol 17(2):187-202

Lynn BH, Abramopoulos F, Avissar R (1995a) Using similarity theory to parameterize mesoscale heat fluxes generated by subgrid-scale landscape discontinuities in GCMs. J Clim 8(4):932-951

Lynn BH, Rind D, Avissar R (1995b) The importance of mesoscale circulations generated by subgrid-scale landscape heterogeneities in general circulation models. J Clim 8(2):191-205

Ma Y, Menenti M, Feddes R, Wang J (2008) Analysis of the land surface heterogeneity and its impact on atmospheric variables and the aerodynamic and thermodynamic roughness lengths. J Geophys Res (Atmos) 113(D08113):1-11

Mahrt L (2000) Surface heterogeneity and vertical structure of the boundary layer. Boundary-Layer Meteorol 96(1-2):33-62

Mailhot J, Benoit R (1982) A finite-element model of the atmospheric boundary layer suitable for use with numerical weather prediction models. J Atmos Sci 39(10):2249-2266

Mason PJ (1988) The formation of areally-averaged roughness lengths. Q J R Meteorol Soc 114(480):399-420

Mellor GL, Yamada T (1982) Development of a turbulence closure model for geophysical fluid problems. Rev Geophys 20(4):851-875

Molod A, Salmun H (2002) A global assessment of the mosaic approach to modeling land surface heterogeneity. J Geophys Res (Atmos) 107(D14):ACL 9-1-ACL 9-18

Molod A, Salmun H, Waugh DW (2003) A new look at modeling surface heterogeneity: extending its influence in the vertical. J Hydrometeorol 4(5):810-825

Mott R, Gromke C, Grünewald T, Lehning M (2013) Relative importance of advective heat transport and boundary layer decoupling in the melt dynamics of a patchy snow cover. Adv Water Resour 55(0):88-97

Mott R, Daniels M, Lehning M (2015) Atmospheric flow development and associated changes in turbulent sensible heat flux over a patchy mountain snow cover. J Hydrometeorol 16(3):13151340

Otto J, Raddatz T, Claussen M (2011) Strength of forest-albedo feedback in mid-holocene climate simulations. Clim Past 7(3):1027-1039

Patton EG, Sullivan PP, Moeng CH (2005) The influence of idealized heterogeneity on wet and dry planetary boundary layers coupled to the land surface. J Atmos Sci 62(7):2078-2097

Polcher J, Laval K, Dümenil L, Lean J, Rowntree P (1996) Comparing three land surface schemes used in general circulation models. J Hydrol 180(1):373-394

Polcher J, McAvaney B, Viterbo P, Gaertner MA, Hahmann A, Mahfouf JF, Noilhan J, Phillips T, Pitman A, Schlosser C, Schulz JP, Timbal B, Verseghy D, Xue Y (1998) A proposal for a general interface between land surface schemes and general circulation models. Glob Planet Change 19(1-4):261-276

Raddatz T, Reick C, Knorr W, Kattge J, Roeckner E, Schnur R, Schnitzler KG, Wetzel P, Jungclaus J (2007) Will the tropical land biosphere dominate the climate-carbon cycle feedback during the twenty-first century? Clim Dyn 29(6):565-574

Raupach M, Finnigan J (1995) Scale issues in boundary-layer meteorology: surface energy balances in heterogeneous terrain. Hydrol Process 9(5-6):589-612

Richtmyer RD, Morton KW (1967) Difference method for initial-value problems, 2nd edn. Interscience Publishers, New York, $405 \mathrm{pp}$

Rieck M, Hohenegger C, van Heerwaarden CC (2014) The influence of land surface heterogeneities on cloud size development. Mon Weather Rev 142(10):3830-3846

Roeckner E, Arpe K, Bengtsson L, Christoph M, Claussen M, Dumenil L, Esch M, Giorgetta M, Schlese U, Schulzweida U (1996) The atmospheric general circulation model echam4: model description and simulation of present-day climate. MPI-Report 218, pp 1-90

Schulz JP, Dümenil L, Polcher J, Schlosser C, Xue Y (1998) Land surface energy and moisture fluxes: comparing three models. J Appl Meteorol 37(3):288-307

Schulz JP, Dümenil L, Polcher J (2001) On the land surface-atmosphere coupling and its impact in a singlecolumn atmospheric model. J Appl Meteorol 40(3):642-663

Segal M, Garratt J, Pielke R, Schreiber W, Rodi A, Kallos G, Weaver J (1989) The impact of crop areas in northeast colorado on midsummer mesoscale thermal circulations. Mon Weather Rev 117(4):809-825

Sellers P (1991) Modeling and observing land-surface-atmosphere interactions on large scales. Surv Geophys 12(1-3):85-114

Sellers P, Mintz Y, Yea Sud, Dalcher A (1986) A simple biosphere model (sib) for use within general circulation models. J Atmos Sci 43(6):505-531 
Seth A, Giorgi F, Dickinson RE (1994) Simulating fluxes from heterogeneous land surfaces: explicit subgrid method employing the biosphere-atmosphere transfer scheme (bats). J Geophys Res (Atmos) 99(D9): 18,651-18,667

Stevens B, Giorgetta M, Esch M, Mauritsen T, Crueger T, Rast S, Salzmann M, Schmidt H, Bader J, Block K, Brokopf R, Fast I, Kinne S, Kornblueh L, Lohmann U, Pincus R, Reichler T, Roeckner E (2013) Atmospheric component of the mpi-m earth system model: echam6. J Adv Model Earth Syst 5(2):146172

Strunin MA, Hiyama T, Asanuma J, Ohata T (2004) Aircraft observations of the development of thermal internal boundary layers and scaling of the convective boundary layer over non-homogeneous land surfaces. Boundary-Layer Meteorol 111(3):491-522

Van den Hurk B, Beljaars A (1996) Impact of some simplifying assumptions in the new ecmwf surface scheme. J Appl Meteorol 35(8):1333-1343

Wieringa J (1976) An objective exposure correction method for average wind speeds measured at a sheltered location. Q J R Meteorol Soc 102(431):241-253

Wood N, Mason P (1991) The influence of static stability on the effective roughness lengths for momentum and heat transfer. Q J R Meteorol Soc 117(501):1025-1056 